\title{
TOWARDS INDUSTRIALISATION OF MICROFLUIDIC SAMPLE-TO-ANSWER SOLUTIONS ENABLING POINT-OF-USE TESTING OF BIOSAMPLES: A DESIGN-FOR-MANUFACTURE LED PLATFORM APPROACH
}

\author{
Jens Ducrée \\ FPC@DCU - Fraunhofer Project Centre for Embedded Bioanalytical Systems at \\ Dublin City University, Ireland
}

\begin{abstract}
The commercial development of many microfluidics-enabled commercial devices requires rapid, optimization and cost-efficient development of competitively priced, and still very rugged, mostly single-use devices. A key challenge remains the seamless scale-up of manufacture from laborious prototyping techniques based on low-throughput machinery and frequently expensive materials to pilot series and industrializable (polymer) mass replication, (bio)functionalization and assembly schemes. Furthermore, the timescales and budgets involved from idea to product are often hard to accept for investors, in particular when considering that many microfluidics-based products will, at least initially, be restricted to comparatively small niche markets or rather tiny fractions of larger markets; hence, economy-of-scale effects are difficult to reach for recovering (sunk) for research and technology development as well as set up of manufacture.

This paper presents a platform approach adopted from many mature industries like automotive or microelectronics for assuring cost-efficient, significantly expedited development of process integrated and automated, microfluidics-enabled solutions at high technology readiness levels (TRLs) with typical application in decentralised "point-of-use" handling and testing of biosamples. Key paradigms supporting this platform approach towards industrialization of microfluidics-enabled solutions are design-for-manufacture (DfM) for seamless scale-up from prototyping to production, quality-by-design (QbD) for robust operation and readiness for scale-up (RfS) towards mass fabrication.
\end{abstract}

\section{INTRODUCTION}

\section{Type of Systems and Industrial Challenge}

Since their inception in the 1980s, microfluidic systems have continuously expanded their space of applications as well as underlying liquid handling and manufacturing technologies [1][2][3][4] $[5][6][7][8][9][10][11]$. A huge variety of solutions has been developed, frequently in the context of the life sciences. This paper focusses on process integrated microfluidic systems that are typically used for comprehensive automation of sample preparation and detection, for instance, in the context of sample handling, preconditioning and / or detection at the point of use. Recent business analysis unanimously confirms that such decentralised solutions constitute a significant fraction of the multi-billion-dollar market for microfluidics-enabled products which is forecast to grow by solid, double-digit figures for the near-to-midterm future [13]. The successful commercialization of such microfluidic components are typically characterised by a set of techno-economical requirements that are common in industrial environments: competitive pricing and performance paired with user-friendliness, reliability during storage, shipping and use as well as seamless immersion with established work flows, technical standards and data management. Depending on the application, and particularly in the arena of medical applications, regulatory compliance presents a major roadblock for successful market introduction.

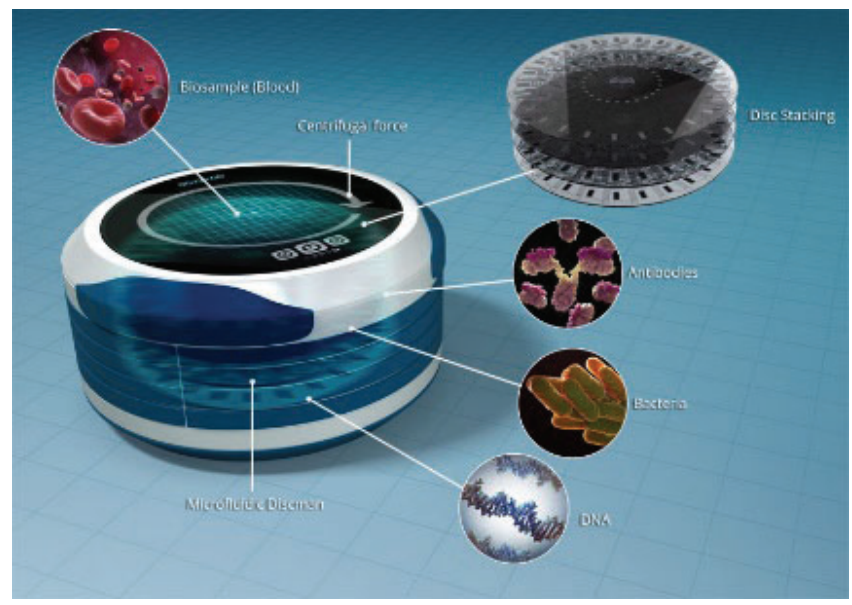

Figure 1: Artist's impression of centrifugal microfluidic "Lab-ona-Disc" platform; a compact and universal instrument containing a spindle motor and detection units can "play" different discshaped polymeric microstructures representing different bioanalytical tests.

\section{Strategies Adopted from Industry and Nature}

Over the last two centuries, industrialization has seen a refinement of its strategies how rather complex technical products like automobiles or computers that were initially exclusive to large corporations and exquisite clientele can be introduced into broader consumer markets. A key enabler of this remarkable transition is tapping into economy-of-scale effects which allows distribution of large investments into the development of product and its production technology such as billion-dollar automotive or semiconductor plants and related marketing budgets over a large number of products sold to consumers. A well-proven recipe for this stage of industrialization is mass customisation based on platform-based approaches.

A key aspect of the second industrial revolution about a century ago was the progression from individual hand-crafting of products from start-to-finish by a small cohort of multi-skill experts to streamlining the underlying technical platform and production processes. In addition to eventually treading the path towards costefficient, high-quality mass manufacturing, this important step was also instrumental towards establishing complex supply chains for components as well as related fabrication equipment and services.

Henry Ford's conveyor-belt factories were a key element of the second industrial revolution where the semi-finished assembly progressively moves through a series of specialised workstations that can be operated by lower-skilled staff. In the third stage, robotics are increasingly replaced by robotic automation. While individual design of derived products was very restricted at the time, a huge repertoire of products can be derived from the same platform, e.g. for chassis or engines in cars, or consumer electronics. 
Notably also nature has chosen to strictly adhere to common blue prints; with some variations and extensions of the theme, nucleic acids store genetic information encoding functional proteins and cells constitute the basic building blocks of higher-level organisms. Amongst animals, quadrupeds possess a basic plot featuring a head containing the brain with two eyes and a central nose above a mouth, linked by a neck to a torso with four extremities. There are of course many more examples of "platforms" or blueprints in biology and industry.

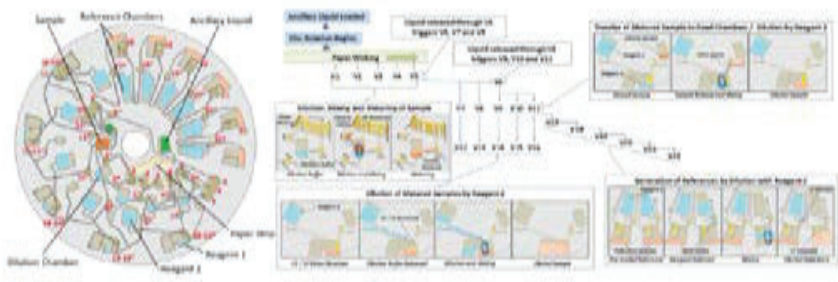

Figure 2: Example for high-level process integration enabled by logical, event-triggered centrifugo-pneumatic flow control on a Lab-on-a-Disc (LoaD) platform. (adopted from [19])

\section{Platform Concept for Industrialization of Microfluidics}

Taking such instructive lessons from mature industries and nature into account, we were looking for a common microfluidic platform that can be customized for automating common liquid handling protocols at the backend of a huge variety of bioanalytical methods at the point-of-use. The main types of these laboratory protocols enable general chemistry, immunoassays and nucleic acid testing as well as cell, bacteria and other bioparticle counting / analysis; our internal market research indeed confirmed a wide range of commercially promising applications in the space of biomedical point-of-care / global diagnostics, liquid handling automation for the life sciences, process analytical techniques and cell line development for biopharma as well as decentralised monitoring the environment, infrastructure, industrial processes and agrifood.

Due to their conceptually simple instrumentation based on a commodity spindle motor without (physical) fluidic interfaces to the typically single-use polymer liquid handling device and the availability of a wide repertoire of robust flow control and sample preparation techniques, we chose the centrifugal microfluidic platform. While such "Lab-on-a-Disc" (LoaD) systems [7][8][9][10][11][12] $[14][15][16][17]$ have been one of the earliest (and still existing) instances of commercially available microfluidic "point-of-care" products, some of them even dating back to market entry in the mid-1990s [1][4], its platform capability has been highlighted only more recently [6].

The recently founded FPC@DCU [18] - the Fraunhofer Project Centre for Embedded Bioanalytical Systems at Dublin City University in Ireland - implements wholistic design-for-manufacture (DfM) and quality-by-design ( $\mathrm{QbD}$ ) paradigms to facilitate rapid and cost-efficient development of high-technology-readiness (TRL) solutions for industry.

\section{STREAMLINED PLATFORM APPROACH}

\section{Centrifugal Liquid Handling}

Testing and handling of biosamples involves a sequence a laboratory unit operations (LUOs) such as sample preparation, reagent addition and mixing / incubation steps. For process automation on microfluidic systems, such protocols are spatio-temporally orchestrated by a sequence of flow control elements such as pumping, valving and routing schemes.

LoaD systems employ the system-innate spindle motor to create a (radial) centrifugal field; due to its strong, square-type scaling, the force field can be modulated over several orders of magnitude by the spin frequency. The rotationally induced artificial gravity conditions also depend on the density of the liquid; this means that handling of (homogeneous) aqueous solutions is quite independent of the given sample and reagents.

Yet, buoyancy effects may be used for stacking layer of (immiscible) media or separating suspended particles possessing specific densities diverging from unity (water). This way often parasitic gas bubbles are suppressed of quickly eliminated from the system. Common batch-wise processing also provides wide independence of flow properties like viscosity which tends to significantly vary in biological samples, thus making the LoaD technology rather robust.

However, the ubiquitous nature of the centrifugal field requires valving schemes to independently control the motion of individual liquid volumes such as sample and multiple reagents that are simultaneously residing on the rotating disc in space and time.

Due to the conceptual difficulty of contact-based interaction from an instrument-based actuation module with a (fast) rotating disc, flow control has often been implemented by variation of the spin rate: high- and low-pass valves open upon transient elevation or reduction of the spin rate, respectively. In the initial state of these typically single-use, normally-closed valves, upstream liquid volumes are held back by counteracting mechanisms such as capillary action, siphoning, sacrificial barriers or opposing pressure differentials, for instance induced by (centrifugal) compression of enclosed gas pockets.

In FPC@DCU, we opted for a new type of centrifugo-pneumatic valving [12][19] based on dissolvable films (DFs) for gating the flow between structures representing LUOs such as metering and mixing that have been developed for performing various tasks in sample preparation.

\section{Platform Approach with Parameterized Elements}

Within the platform approach, integrated microfluidic systems are composed from a library containing functional elements for flow control and LUOs for manipulation of liquid samples. These elements are geometrically parametrized, i.e., they can be flexibly adjusted, within a given range, to requirements like volume capacity, shape or their location on the disc.

\section{Design-for-Manufacture for Seamless Scale-Up}

The functionality of microfluidic systems is tightly linked to the underlying manufacturing schemes. Issues frequently arise from artefacts intrinsic to the specific fabrication and assembly scheme, and even more when switching manufacturing schemes for the same structure during scale-up from prototyping to small series, e.g., for in-field testing, and mass production.

To mitigate the associated development risks, FPC@DCU implements a design-for-manufacture (DfM) paradigm tackling the issue from two sides; manufacturing tolerances which are likely to impact fluidic functionality are thoroughly qualified and minimized by process optimization. In addition, fluidic elements are designed as forgiving as possible for (unavoidable) statistical deviations in manufacturing processes as well as properties of structural (solid) materials, surfaces and liquids.

\section{Virtual Prototyping for Efficient Development}

FPC@DCU's platform approach is supported by fluidic simulation following a strategy that has been termed in other contexts as virtual prototyping or the creation of a digital twin. For the complex, multi-step, multi-phase and multi-branched systems considered here, a full system-level computational fluidic dynamic si- 
mulation is often not practical. However, we can make use of the fact that the overwhelming majority of centrifugal microfluidic systems operate in a batch-wise fashion where the individual stages between sample preparation and detection are interspersed by valves and thus hydrodynamically widely decoupled from each other.

This approximate hydrodynamic isolation allows to decompose the network into individual functional units which, in a first step, can be modelled and simulated separately. Transfer functions equivalent to resistors, capacitances, inductances and diodes in electric circuits may be defined, either through analytical formulas or from numerical solutions and their fits. In a lumped-element approach, these separate elements are then linked to create the fluidic networks for automating full assay protocols while massively reducing the numerical complexity and computational requirements for simulation.

On the one hand, such virtual "in silico" prototyping and testing of individual elements may reveal fundamental working principles, thus helping to identify critical parameters and the impact of deviations in geometrical structure and liquid (input) volumes. On the other hand, the "lumped-element" modelling also allows to flexibly configure networks enabling new applications from a set of validated, well-understood library elements, thus significantly expediting and de-risking the development of novel, hightechnology-readiness-level (TRL) applications.

\section{Quality-by-Design for Robust Functionality}

The library of functional fluidic elements is characterized by several (quantitative) figures of merit which, for instance, comprise of the geometry-dependent range and standard deviation for centrifugal burst frequencies, actuation / response times and transferred volumes.

Towards microfluidic system integration needed for multi-step sample preparation, error propagation has a tremendous impact on final results, e.g., on the definition of (preconditioned) sample and reagent volumes to be mixed prior to a final detection step. As a specific feature of centrifugal microfluidic systems, also the overall functionality of liquid handling is impacted by the accuracy of volume definition in the preceding steps. This is because the centrifugally induced field translates, via the mean radial position and extension of the liquid "plug", into the (hydrostatic) pressure governing the transport of the liquid.

Consequently, any volume inaccuracies inflicted in an upstream liquid handling step influence the hydrodynamic behavior of succeeding operations, possibly even leading to malfunction on a system level. Such volume errors might be attributed to the initial loading of the disc by pipetting as well as manufacturing tolerances affecting channel cross sections and thus modulating the radial location and extensions of residual liquid plugs which set the centrifugal force.

FPC@DCU's Quality-by-Design (QbD) strategy pursues a twopronged approach to increase the robustness of operation. On the one hand, the precision of manufacturing processes is optimized to minimize geometrical deviation in all stages of development from prototyping to mass manufacture (see above). On the other hand, simulation coupled with experimental validation allows to design fluidic elements with maximum tolerance to fluctuations in incoming liquid volumes.

\section{CONCLUSIONS \& OUTLOOK}

This paper has described a path how to accelerate and de-risk research and technology development of microfluidics-enabled solutions delivering decentralised testing of biosamples engineered for point-of-use applications in the context of the life-sciences. High TRLs will be accomplished by a combination of strategies: a streamlined platform approach based on a library of validated, geometrically parametrized fluidic modules to enable flexible configuration of new networks, design-for-manufacture leveraging quasi seamless scale-up towards mass fabrication, virtual prototyping / digital twin to accelerate functional optimization and quality-by-design to assure error tolerance at the system level.

All these principles are advanced in FPC@DCU and its partner, the Fraunhofer Institute for Production Technology, to offer a viable path towards successful contract development of microfluidics-enabled devices for biomedical point-of-care / global diagnostics, liquid handling automation for the life sciences, process analytical techniques and cell line development for biopharma as well as decentralised monitoring the environment, infrastructure, industrial processes and agrifood.

\section{ACKNOWLEDGEMENTS}

This publication has emanated from research conducted with the financial support of Science Foundation Ireland (SFI) and Fraunhofer-Gesellschaft under the SFI Strategic Partnership Programme, Grant Number 16/SPP/3321.

\section{REFERENCES}

[1] C. T. Schembri, V. Ostoich, P. J. Lingane, T. L. Burd and S. N. Buhl, Clin. Chem., 38, 1665-70 (1992).

[2] M. J. Madou M J and G. J. Kellogg, Proc. SPIE 3259 80-93 (1998).

[3] M. Madou, J. Zoval, G. Jia, H. Kido, J. Kim and N. Kim, Annu. Rev. Biomed. Eng., 8 601-28 (2006).

[4] M. Gustafsson, D. Hirschberg, C. Palmberg, H. Jörnvall and T. Bergman, Anal. Chem. 76 345-350 (2004).

[5] M. Inganäs, H. Dérand, A. Eckersten, G. Ekstrand, A.-K. Honerud, G. Jesson, G. Thorsén G, T. Söderman and P. Andersson, Clin. Chem., 51 1985-7 (2005).

[6] Jens Ducrée and Roland Zengerle. FlowMap - Microfluidics roadmap for the life sciences. Books on Demand $\mathrm{GmbH}$, Norderstedt, Germany (2004).

[7] J. Ducrée, S. Haeberle, S. Lutz, S. Pausch, F. von Stetten, R. Zengerle, J. Micromech. Microeng., 17, S103-S115 (2007).

[8] D. Mark, S. Haeberle, G. Roth, F. von Stetten and R. Zengerle, Chem. Soc. Rev., 39, 1153-1182 (2010).

[9] O. Strohmeier, M. Keller, F. Schwemmer, S. Zehnle, D. Mark, F. von Stetten, R. Zengerle, N. Paust, Chem. Soc. Rev. 44, 6187-6229 (2015).

[10] M.C.R. Kong, E.D. Salin, Anal. Chem., 84(22): 10038-10043 (2012).

[11] S. Smith et al., Micromachines, 7(2), pp. 22 (2016).

[12] R. Gorkin, C.E. Nwankire, J. Gaughran, X. Zhang, G.G. Donohoe, M. Rook, et al., Centrifugo-pneumatic valving utilizing dissolvable films, Lab Chip, 12 2894-902 (2012).

[13] Yole Développement, Status of the Microfluidics Industry (2017).

[14] Beom Seok Lee, Yang Ui Lee, Han-Sang Kim, Tae-Hyeong Kim, Jiwoon Park, Jeong-Gun Lee, Jintae Kim, Hanshin Kim, Wee Gyo Lee, Yoon-Kyoung Cho, Lab Chip 11(1), 70-78 (2011).

[15] Liviu Clime, Daniel Brassard, Matthias Geissler and Teodor Veres, Lab Chip, 15, 2400-2411 (2015).

[16] Brandon L. Thompson, Christopher Birch, Daniel A. Nelson, Jingyi Li, Jacquelyn A. DuVall, Delphine Le Roux, An-Chi Tsuei, Daniel L. Mills, Brian E. Root and James P. Landers, Lab Chip, 16, 4569-4580 (2016).

[17] D. J. Kinahan, S. M. Kearney, N. Dimov, M. T. Glynn, J. 
Ducrée, Lab Chip, 14, 2249-58 (2014).

[18] FPC@DCU - Fraunhofer Project Centre for Embedded Bioanalytical Systems at Dublin City University, Ireland, www.dcu.ie/fpc.

[19] David Kinahan, Sinéad Kearney, Olivier Faneuil, Macdara

Glynn, and Jens Ducrée. RSC Advances, 5(3):1818-1826 (2015).

CONTACT

* Jens Ducrée, tel: +353-1-700-7658; jens.ducree@dcu.ie 